\title{
Age-Related Differences in Random Generation
}

\author{
Martial Van der Linden, ${ }^{*} \dagger$ Annick Beerten, $\dagger$ and Mauro Pesenti $\dagger$ \\ *Neuropsychology Unit, University of Liège, Liège, Belgium and †Cognitive \\ Neuropsychology Research Unit, University of Louvain, Louvain-la-Neuve, Belgium
}

This study investigated the effects of age on a random generation task. In Experiment 1 , young and elderly subjects were asked to generate random strings of letters at 1-, 2-, and 4-s rates. The elderly subjects produced more alphabetical stereotype responses than young subjects, even in the slowest rate condition. Furthermore, as faster rates were imposed, elderly subjects could no longer maintain the pace and missed responses. In Experiment 2, subjects were required to generate letters at the same time that they sorted cards into one, two, four, or eight categories. Age-related differences were observed on most of the measures of randomness (stereotypes, zero-order, and first-order measures). In addition, the number of errors increased with the number of sorting alternatives, especially for elderly subjects. These results suggest the existence of a reduction of the central executive resources, along with a reduced inhibition ability, in the elderly subjects. However, the contribution of a perceptual speed factor is also discussed. () 1998 Academic Press

According to Baddeley $(1986,1996)$, attempting to generate random sequences of items is an activity that places significant demands on the central executive component of working memory. Indeed, when subjects are required to produce random sequences of letter names or digits, they have to select new strategies to keep the sequence as random as possible, prevent the occurrence of schematic responses (e.g., alphabetic stereotypes, such as $L M N$ ), check that the responses are suitably random, and, if not, change the strategy. All these selection and control functions correspond exactly to the role that is assigned by Baddeley (1986) to the central executive system.

In a random generation study conducted on young adults, Baddeley (1966) showed that redundancy and stereotype responses increased linearly with rate (Experiment 1). In addition, when subjects are required to generate random sequences at the same time that they are performing a card-sorting task, the

Address reprint requests to Martial Van der Linden, Service de Neuropsychologie, Boulevard du Rectorat B33, Sart Tilman, 4000 Liège, Belgium. Electronic mail may be sent via Internet to mvanderlinden@ulg.ac.be. The research was supported by the "Action de Recherche Concertée 92/97-158 du Ministère de la Communauté Française de Belgique'. We thank Vincent Yzerbyt for his helpful statistical advice. 
redundancy of the responses increased linearly with the sorting load (Experiment 2). In the same vein, Spence, Wolitsky, and Pezenik (1969) showed that instructions to recall rather than listen to passages of words varying in their approximation to English while subjects were simultaneously generating random sequences produced a significant decrement in randomness. Furthermore, the decrease varied inversely with the amount recalled. These results suggest that the ability to generate random sequences depends on a response-selection mechanism of limited capacity: randomness decreases when subjects have less time or fewer resources at their disposal. More generally, these data indicated that random generation might be a good task to explore both the capacity and efficiency of the central executive component of working memory.

In an anecdotal way, Baddeley (1986) reported that some years ago, when returning home for Christmas, he administered a random generation task to numerous aunts and uncles, using cousins as their controls. He found a clear age effect, with some of the more elderly producing a high proportion of stereotyped responses. Wiegersma and Meertse (1990) also observed that the performance of older adults was reduced relative to younger subjects in a randomization span task which required the production of all numbers of a set $(1-3,1-4$, etc.) in a self-chosen ordering, with the explicit instruction to avoid counting order and not to produce any of the numbers twice. On the other hand, no differences between age groups were observed in a forward digit span task and a missing scan in which the subjects were asked to detect the missing item in random sequences of $n-1$ numbers. More recently, Daigneault, Braun, and Whitaker (1992; see also Daigneault \& Braun, 1993, and Shimamura \& Jurica, 1994) also observed a significant age effect on the Self-Ordered Pointing Task (SOPT), which closely resembled the randomization span task devised by Wiegersma and Meertse (1990). The SOPT has been used as an index of frontal lobe dysfunction (Petrides \& Milner, 1982). This age effect on the SOPT has been interpreted in terms of a deficit of frontal origin and affecting inhibitory control or monitoring within working memory.

A number of studies have also examined random generation in neuropsychological patient groups, such as patients suffering from a dysexecutive syndrome due to a chronic frontal lesion or to Parkinson's disease (Spatt \& Goldenberg, 1993), survivors of severe closed-head injury (Azouvi, Jokic, Van der Linden, Marlier, \& Bussel, 1996), patients suffering from an episode of transient global amnesia (Brugger, Landis, \& Regard, 1992), or patients with dementia of the Alzheimer type (Brugger, Monsch, Salmon, \& Butters, 1996). All these studies showed that sequences generated by brain-damaged patients were less random than those generated by their respective control group. In addition, a few studies suggest that different cognitive deficits are associated with qualitatively different response patterns (for a review, see Brugger et al., 1996). More specifically, it appears that the main difference between patients with frontal lobe dysfunction and control subjects was the 
persistence of a single production strategy (counting; e.g., Spatt \& Goldenberg, 1993), most likely due to a difficulty to inhibit overlearned schemas. In the same perspective, Brugger et al. (1996) showed that random sequences generated by patients with dementia of the Alzheimer type (DAT) were more stereotyped (contained fewer digit combinations) than those of elderly normal control subjects and that this enhanced response stereotype was mainly due to an overrepresentation of ordinal sequencing (counting bias). Finally, the degree of sequential nonrandomness exhibited by the DAT patients was positively correlated with severity of impairment in a variety of neuropsychological tests which assess the efficiency of executive functions, and more specifically the efficiency of frontal lobe-mediated generation processes. On the other hand, a failure to avoid repetitions has been observed in patients suffering from an episode of transient global amnesia (Brugger et al. 1992). This deficit was interpreted as suggesting the existence of a deficit affecting the hippocampal inhibitory processes. Alternatively, this failure to avoid repetitions might have a memory basis since it only appeared when a slow generation rate was required ( 1 random number per $5 \mathrm{~s}$ ) and not at a rate of one per second.

The purpose of the experiments reported here was to examine further the effect of age on random generation. More specifically, we investigated in young and elderly subjects the effect of different generation rates and of a simultaneous second task on random generation. In Experiment 1, the young and elderly subjects' task was to generate random string of letters at 1-, 2-, and 4-s rates. In Experiment 2, subjects were required to generate letters at the same time that they were sorting cards into one, two, four, or eight categories at a 2-s rate. If random generation depends on a limited capacity central executive system and if, as suggested in some studies (e.g., Van der Linden, Brédart, \& Beerten, 1994), elderly subjects show a reduced capacity of this system, then random generation performance of older adults should be affected, particularly as faster generation rates are imposed and more complex sorting tasks are introduced. Furthermore, inasmuch as neuropathological, neuroanatomical, and neurophysiological studies as well as studies of cognitive aging using neuropsychological tests indicate the role of frontal dysfunction in some aspects of age-related differences in cognition (see Braun \& Lalonde, 1990; Daigneault et al., 1993; Moscovitch \& Winocur, 1992; West, 1996), it was hypothesized that elderly subjects would exhibit an excessive number of stereotyped responses, which seem to be typically associated with frontal dysfunction (e.g., Spatt \& Goldenberg, 1993).

\section{EXPERIMENT 1}

\section{Method}

Subjects. Subjects were 16 young adults and 16 elderly subjects. Young subjects ( 8 males and 8 females) were students at the Louvain-la-Neuve University. They were aged between 20 and 30 years (mean age: 23.2). Their mean Mill Hill score (French language adaptation 
of the multiple choice synonym subtest, Deltour, 1993) was $27.4(S D=3.84)$, and they had completed between 12 and 16 years of schooling. The elderly subjects ( 8 males and 8 females) were recruited from the Louvain-la-Neuve Senior University. They were healthy people living in the community and aged between 60 and 70 years (mean age: 66.1). They had completed 12 or more years of schooling. Their mean score on the Mill Hill Vocabulary test was 30.4 $(S D=2.55)$. The vocabulary scores of the elderly were significantly higher than those of the young subjects $(t(30)=2.66, p<.05)$.

Procedure. A random generation task similar to the one used by Baddeley (1966, Experiment 1) was performed by young and older adults. The subjects were required to call out a sequence of 100 letters from the alphabet at each of three rates paced by bip tones-one letter per 1, 2 and $4 \mathrm{~s}$. The order of presentation of conditions was randomized with the constraint that no subject began with the 1-s rate. Similar to Baddeley (1966), they were told to imagine that they were drawing letters from a hat one at a time, calling them out, then replacing them, so that on each draw any of the 26 letters of the alphabet was equally likely to be selected. It was pointed out explicitly that the sequence would be completely jumbled and would not therefore be likely to comprise French words or alphabetic sequences such as $A B C$ or $X Y Z$.

The following measures of randomness were used: (1) a stereotype measure based on the frequency with which the subject produced digrams which follow the alphabetical stereotypes (e.g., $A B, K L$, or $Y Z$; see Baddeley, 1966; Spatt \& Goldenberg, 1993); (2) a repair measure based on the frequency with which the subject produced immediate repetitions (e.g., $A A$ or $D D$, see Spatt \& Goldenberg, 1993); (3) a zero-order redundancy measure based on the relative frequency with which individual letters were used: the more the frequency with which individual letters is unbalanced, then the more the sequence is redundant. Details of the algorithm for computation of the zero-order redundancy measure may be found in Baddeley (1966; see also Attneave, 1959). This measure varies from 0 to 1 ; the closer it is to 1 , the higher the nonrandomness; and (4) a first-order redundancy measure (RNG index; Evans, 1978) which measures the difference between expected and observed probabilities of pairs of letters. Details of the algorithm for computation of RNG are described by Evans (1978). This index varies from 0 to 1 ; the closer it is to 1 , the less random the sequence.

\section{Results}

Table 1 shows the averaged scores for each measure, according to group and rate. Comparison between young and older subjects was performed using a $2 \times 3$ doubly multivariate analysis of variance (Tabachnick \& Fidell, 1989), taking age as a between-subjects and rate as a within-subjects factor. Four dependent variables (DVs) were used: zero-order redundancy, stereotypes, RNG index, and missed responses. The observed frequency of repairs being very low in both groups, this measure was not included in the statistical analysis.

For the set of combined DVs, the effects of age, rate, and their interaction were all significant: young subjects produced more random sequences [Wilks' lambda: .66; $F(4,27)=3.5, p<.02$ ], randomness decreased as rate increased [Wilks' lambda: $.15 ; F(8,23)=15.8, \mathrm{p}<.0001$ ], and the departure from randomness was higher for older subjects than young subjects as rate increased [Wilks' lambda: .53; $F(8,23)=2.6, p<.04$ ]. A careful look at this interaction showed that the two groups significantly differed at the fastest rate [Wilks' lambda: .6; $F(4,27)=4.5, p<.007$ ] but not at the 
TABLE 1

Mean Scores (and SD) for All Measures According to Age and Rate in Experiment 1

\begin{tabular}{|c|c|c|c|c|c|c|c|}
\hline \multirow[b]{2}{*}{ Group } & \multirow[b]{2}{*}{ Measures } & \multicolumn{6}{|c|}{ Rate } \\
\hline & & \multicolumn{2}{|c|}{$1 \mathrm{~s}$} & \multicolumn{2}{|c|}{$2 \mathrm{~s}$} & \multicolumn{2}{|c|}{$4 \mathrm{~s}$} \\
\hline \multicolumn{8}{|c|}{ Young $(N=16)$} \\
\hline & Redundancy & 0.075 & $(0.03)$ & 0.05 & $(0.02)$ & 0.03 & $(0.01)$ \\
\hline & Stereotypes & 13.37 & $(5.62)$ & 8.06 & $(4.45)$ & 4.0 & $(2.9)$ \\
\hline & RNG index & 0.14 & $(0.05)$ & 0.10 & $(0.03)$ & 0.08 & $(0.02)$ \\
\hline & Repairs & 0.56 & $(0.73)$ & 0.25 & $(0.58)$ & 0.12 & $(0.05)$ \\
\hline & Missed responses & 0 & & 0 & & 0 & \\
\hline \multicolumn{8}{|c|}{ Elderly $(N=16)$} \\
\hline & Redundancy & 0.07 & $(0.02)$ & 0.06 & $(0.03)$ & 0.04 & $(0.02)$ \\
\hline & Stereotypes & 19.8 & (14.7) & 11.9 & $(6.5)$ & 6.0 & $(5.5)$ \\
\hline & RNG index & 0.17 & $(0.09)$ & 0.11 & $(0.04)$ & 0.08 & $(0.03)$ \\
\hline & Repairs & 0.25 & $(0.45)$ & 0.56 & (1.03) & 0.06 & $(0.25)$ \\
\hline & Missed responses & 4.7 & $(5.9)$ & 2.1 & $(3.7)$ & 0.12 & $(0.3)$ \\
\hline & Redundancy & 0.07 & $(0.02)$ & 0.06 & $(0.03)$ & 0.04 & $(0.02)$ \\
\hline & Stereotypes & 16.6 & (11.4) & 10.0 & $(5.9)$ & 5.0 & $(4.5)$ \\
\hline & RNG index & 0.15 & $(0.07)$ & 0.11 & $(0.04)$ & 0.08 & $(0.03)$ \\
\hline & Repairs & 0.41 & $(0.61)$ & 0.41 & $(0.84)$ & 0.09 & $(0.39)$ \\
\hline & Missed responses & 2.3 & $(4.8)$ & 1.03 & $(2.8)$ & 0.06 & $(0.25)$ \\
\hline
\end{tabular}

2-s rate [Wilks' lambda: .8; $F(4,27)=2.2, p<.1$ ] nor at the 4-s rate [Wilks' lambda: $.8 ; F(4,27)=1.9, p<.2]$.

When considering each individual measure, a stepdown analysis of variance $^{1}$ showed that the effect of age was significant for stereotypes [stepdown $F(1,29)=4.1, p<.05$ ] and missed responses [stepdown $F(1,27)=7.1$, $p<.02$ ], but not for zero-order redundancy [stepdown $F(1,30)=0.55$; $p<.5$ ] and the RNG index [stepdown $F(1,28)=0.99, p<.4$ ]. The effect of rate was highly significant for zero-order redundancy [stepdown $F(2,60)$ $=29.3, p<.0001$ ], stereotypes [stepdown $F(2,59)=21.1, p<.0001$ ], and missed responses [stepdown $F(2,57)=5.1, \mathrm{p}<.001$ ], but not for the

${ }^{1}$ A univariate analysis was also performed to look for any further information. Some of the DVs were however slightly correlated: pooled within-cell correlations among DVs ranged from -.18 to .15 , except for stereotypes which were correlated with the RNG index (pooled correlation: .68) and with missed responses (pooled correlation: - .39). For that reason, a stepdown analysis of variance was preferred (Bock \& Haggard, 1968; Bray \& Maxwell, 1985; Tabachnick \& Fidell, 1989). In this stepdown analysis, priorities were assigned to DVs and each DV was analyzed, in turn, with the higher-priority DVs treated as covariates and the highest-priority DV tested in a univariate ANOVA. A somewhat arbitrary ordering of DVs was used here because DVs could not be easily prioritized on theoretical grounds. Both the univariate and the stepdown analysis of variance led to the same pattern of results. The reader will find the summary of results of both analysis, as well as the pooled within-cell correlations among DVs, in the appendix. 
RNG index ${ }^{2}$ [stepdown $F(2,58)=1.8, p<.2$ ]. Post-hoc analyses using paired $t$-tests indicated that for each measure, all rate conditions were significantly different from each other (all observed $p$ at least $<.05$ ). Finally, the interaction between age and rate was significant only for missed responses [stepdown $F(2,57)=12.2, p<.0001$ ].

Because the subjects had to generate random series several times in a row, data were examined for possible practice or tiredness effects. The departure from randomness was examined at each single rate according to the order of occurrence of that rate in the experimental session (for example, the 2-s rate could be the first, the second or the third condition performed by the subject). No effect on the order of occurrence within the session was observed at the 1- and 4-s rate conditions. At the 2-s rate, randomness decreased when this condition appeared as the second one (Wilks' lambda: $41 ; F(8$, $46)=3.14, p<.007$ ) but not as the last one (and this only for zero-order redundancy and RNG index). The order of occurrence never interacted with age.

\section{Discussion}

Experiment 1 revealed the effects of age, rate and their interaction for the set of combined DVs. There was a clear relationship between rate and deviation from randomness: the faster the rate, the higher the nonrandomness as measured by zero-order redundancy, stereotypes, and missed responses. Moreover, elderly subjects produced less random sequences than young subjects. They mainly produced less random sequences than young subjects. They mainly produced more alphabetical stereotypes than young subjects, and this was observed even at the slowest rate condition (one response every $4 \mathrm{~s}$ ) because there was no age $\times$ rate interaction; they also often missed responses, which young subjects never did. Furthermore, at the faster rates of one item per 1 and $2 \mathrm{~s}$, the task appeared to place excessive strain on the capacity of the elderly subjects' central executive, which led to more and more missing responses.

No practice nor tiredness effects were observed in this experiment (we have no explanation for the effect observed at the 2-s rate condition, but this clearly does not fit with practice or tiredness effects). This result confirms the absence of practice effect across repeated trials of random generation found by Evans (1978).

The second experiment was carried out to explore the ability of young

${ }^{2}$ However, the univariate analysis revealed a significant effect for this measure (see appendix). This can probably be explained by the fact that the RNG index was correlated with the stereotypes which already accounted for the overlapping variance. Note that if the RNG index is introduced before stereotypes in the ordering of DVs, then rate appears to be significant for all measures (including the RNG index). 
and elderly subjects to generate random sequences when a part of the central executive's resources was allocated to a simultaneous card sorting task.

\section{EXPERIMENT 2}

\section{Method}

Subjects. The same 32 subjects who participated in Experiment 1 were tested. No practice effects were expected in this experiment given that Evans (1978) did not observe practice effects across repeated trials of random generation (see also the absence of practice effects in Experiment 1).

Procedure. A sorting and random generation task similar to the one used by Baddeley (1966; Experiment 2) was performed by young and older adults. The subjects were asked to call out a sequence of 100 letters from the alphabet at a constant 2-s rate. Simultaneously, they were required to sort packs of 100 playing cards into one, two (kings and eights), four (aces, twos, threes, fours), or eight (aces, twos, threes, fours, fives, sixes, eights and nines) categories. The one-choice situation empoyed one of the multichoice packs with the subjects instructed to ignore card values; they just had to pick up cards and place them one upon each other at a paced rate. The cards were held face down and sorted with the preferred hand into a sorting frame consisting of eight compartments, each open at the end facing the subjects. When in use, each compartment contained an appropriate marker card. The lowest card value was always on the left and the highest on the right. Each subject performed the four sorting conditions in a different random order. The instructions for random generation were similar to those proposed in Experiment 1. All subjects performed Experiment 2 after Experiment 1.

\section{Results}

Table 2 shows the averaged scores for each measure, according to group and number of sorting alternatives. Comparison between young and older subjects was performed using a $2 \times 4$ doubly multivariate analysis of variance, taking age as a between-subjects and number of sorting alternatives as a within-subjects factor. Five DVs were used: the same four DVs as in Experiment 1 (zero-order redundancy, stereotypes, RNG index, and missed responses) to which the sorting errors scores were added. Again, the observed frequency of repairs being very low in both groups, this measure was not included in the statistical analysis.

For the set of combined DVs, the effects of age, number of sorting alternatives and their interaction were all significant: young subjects produced more random sequences (Wilks' lambda: .31; $F(5,26)=11.5, p<.0001$ ), randomness decreased as the number of sorting alternatives increased (Wilks' lambda: .08; $F(15,16)=12.7, p<.0001)$, and the departure from randomness was higher for older subjects than young subjects as the number of sorting alternatives increased (Wilks' lambda: .15; $F(15,16)=5.8, p<$ $.001)$. A careful look at this interaction showed that the two groups significantly differed at each condition of sorting [one choice: $F(4,27)=2.7$, $p<.05$; two choices: $F(5,26)=3.9, p<.01$; four choices: $F(5,26)=$ 9.1, $p<0001$; eight choices: $F(5,26)=17.3, p<.0001]$.

When considering each individual measure, a stepdown analysis of vari- 


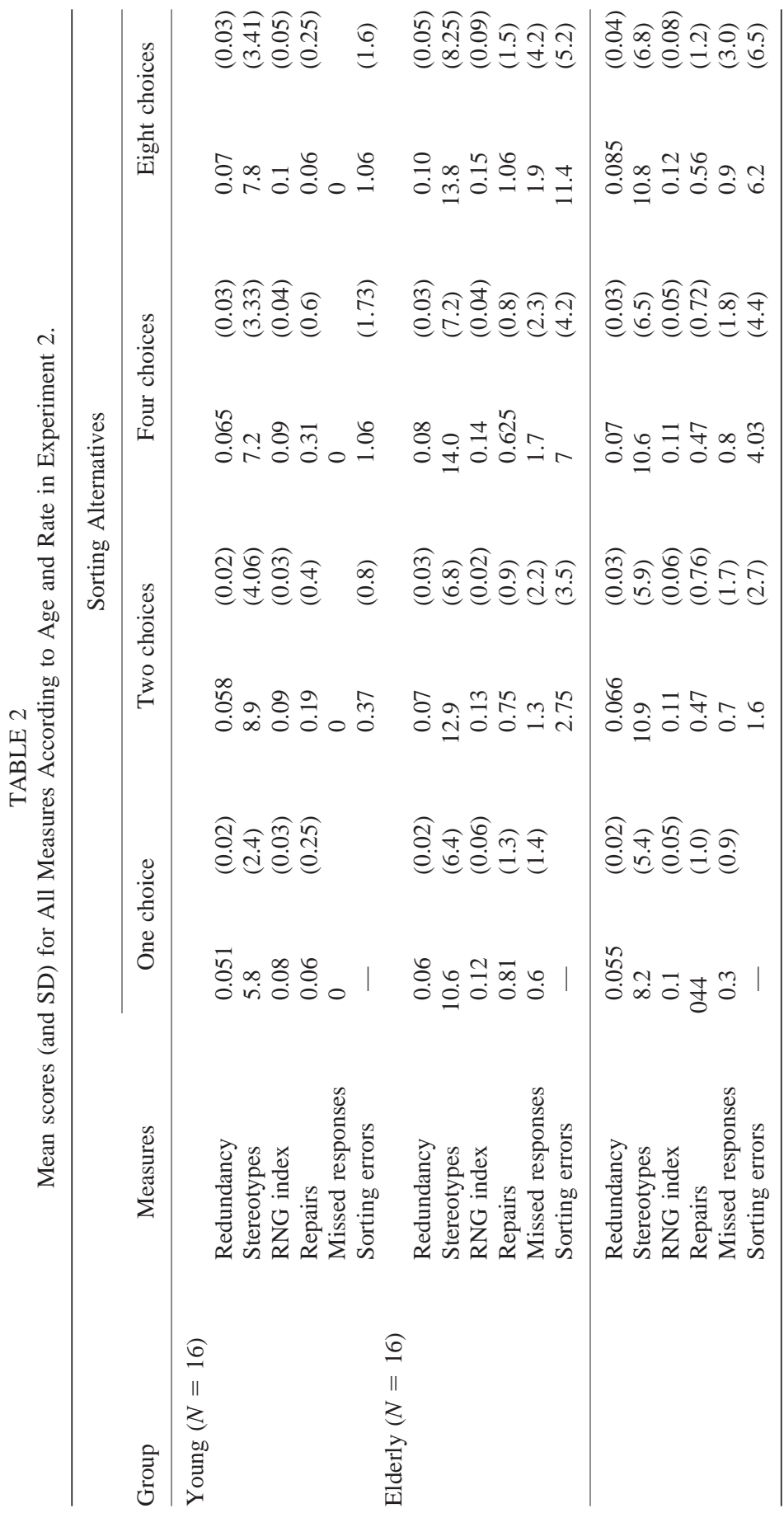


ance $^{3}$ showed that the effect of age was significant for zero-order redundancy [stepdown $F(1,30)=4.3, p<.05$ ], stereotypes [stepdown $F(1,29)=10.1$; $p<.005$ ], and sorting errors [stepdown $F(1,26)=25.8, p<.0001$ ], but not for the RNG index [stepdown $F(1,28)=0.04, p>.9$ ] and missed responses [stepdown $F(1,27)=1.2, p<.3$ ]. The effect of the number of sorting alternatives was highly significant for zero-order redundancy [stepdown $F(3,90)=9.7, p<.0001$ ], stereotypes [stepdown $F(3,89)=3.8$, $p<.02$ ], and sorting errors [stepdown $F(3,86)=39.7, p<.0001$ ], but not for the RNG index [stepdown $F(3,88)=0.04, p<.2$ ] and missed responses [stepdown $F(3,87)=2.1 ; p<.2$ ]. Post-hoc analyses, using paired $t$-tests (all observed $p$ at least $<.05$ ), indicated that for zero-order redundancy, the one-choice condition significantly differed from all the other choice conditions and the two-choice condition differed from the eight-choice condition. With regard to stereotypes, the one-choice condition significantly differed from all the other conditions. The interaction between age and rate was significant only for sorting errors [stepdown $F(3,86)=33.7 ; p<.0001$ ]. Posthoc analyses, using paired $t$-tests (all observed $p$ at least $<01$ ), indicated that in the older group, all choice conditions were significantly different from each other, whereas in the young group, the number of errors differed only between the one-choice and two-choice conditions and the eight-choice conditions.

Data were examined for possible practice or tiredness effects using the same procedure as in Experiment 1. The order of occurrence of any choice condition within the session never affected randomness, and it never interacted with age.

\section{Discussion}

Experiment 2 revealed clear age-related differences in random generation performance when a simultaneous card-sorting task was introduced. These age differences were observed for most of the measures of randomness (firstorder redundancy, alphabetical stereotypes, and sorting errors). Moreover, the deviation from randomness increased with the number of sorting alternatives. Again, this was observed for the first-order redundancy, the number of alphabetical stereotypes, as well as for the number of sorting errors. Finally, the effect of the number of sorting alternatives was more pronounced for elderly subjects than for young subjects. As the number of sorting alternatives increased, the elderly subjects produced more and more sorting errors. Again, no practice nor tiredness effects were observed.

\footnotetext{
${ }^{3}$ See note 1 . Both the univariate and the stepdown analysis of variance led to the same pattern of results. The reader will find the summary of results of both analyses, as well as the pooled within-cell correlations among DVs, in the appendix.
} 


\section{GENERAL DISCUSSION}

Experiment 1 revealed age-related decrements in random generation performance. Decrements were observed even in the slowest rate condition (4-s rate) for some measures. Indeed, the elderly subjects produced more alphabetical stereotype responses than young subjects; furthermore, as faster rates were imposed, random generation became too demanding for elderly subjects, and they could no longer maintain the pace and missed responses. In Experiment 2, when a card-sorting task was introduced simultaneously with random generation, age-related differences were observed on most of the measures of randomness (first-order redundancy, alphabetical stereotypes, and sorting errors). In addition, as the complexity of the card-sorting task increased, older subjects appeared to give priority to random generation to the detriment of card sorting, which led to more and more sorting errors.

It remains to identify the nature of the age-related differences observed in both experiments. According to Spatt and Goldenberg (1993), there are three possible sources of deviation from randomness. First, in a random generation task, subjects have to produce random sequences and to change from one strategy to another when the responses become less random. The execution of these steps will depend on the availability of alternative production strategies, the ease to rapidly shift from one procedure to another, and the ability to inhibit overlearned procedures (e.g., alphabetical stereotypes, such as $A B C$ ). Second, nonrandomness may be partly due to the fact that subjects are guided by a wrong concept of randomness. As a matter of fact, it appears that a difference between the human concept of randomness and actual or computer-simulated randomness is that human subjects tend to avoid immediate repetitions in random production (see Evans, 1978; Wiegersma, 1982; Spatt \& Goldenberg, 1993, Experiment 1). Furthermore, human subjects tend to confound randomness with evenness and, consequently, human productions are more even and therefore less redundant than actual or computersimulated random sequences (Spatt \& Goldenberg, 1993, Experiment 1). Third, if subjects identify randomness with evenness, they should be able to monitor the evenness of occurrence of single letters or digits as far as the sequences of items can be kept in working memory (i.e., sequences of less than 10 items). On the other hand, the capacity of working memory is not sufficient to monitor the distribution of pairs of items since the expected frequency of any pair of items is very low.

In this theoretical framework, the existence of an age-related difference in the zero-order redundancy (a measure based on the relative frequency with which individual letters were used) should indicate a reduced monitoring ability or even a reduced short-term storage capacity. On the other hand, age-related variations in the first-order redundancy measure (the RNG index which measures the difference between expected and observed probabilities of pairs of letters) might not be interpreted as an indication of reduced moni- 
toring (or short-term storage capacity) since monitoring for pairs exceeds the capacity of working memory even in young adults. Rather, it should suggest a reduced ability to shift from one response schema to another or a limited availability of production policies (see Spatt \& Goldenberg, 1993). Finally, an excessive number of stereotyped responses could indicate a difficulty to inhibit overlearned schemas.

Wiegersma (1982) also proposed that when subjects are asked to produce a random sequence of responses, they try to control perseveration tendancies and stereotyped orders (which come to mind because of their memory strength) by the use of a control mechanism which compares each candidate response with the last seven responses, stored in short-term memory, and rejects the candidate if it is found among them. According to Wiegersma, a decreased control of strength-determined tendencies could be invoked to explain the effects of a simultaneous second task on random generation, i.e. the increase of stereotyped responding (Evans, 1978; Baddeley, 1966) and a decrease of repetition avoidance (Wagenaar, 1972).

With regard to the present study, the lack of any difference between young and elderly subjects in zero-order and first-order measures (in Experiment 1) indicates that the concept of randomness and availability of alternative production schemata did not differ between both groups. In addition, this also suggests that older adults' monitoring, shifting and short-term storage abilities were not significantly reduced. However, the fact that elderly subjects produced more alphabetical stereotypes (even in the slowest rate condition) suggests that the major source of nonrandomness in these subjects was a difficulty to inhibit routine procedures. This difficulty to inhibit overlearned routines appeared even more pronounced when faster rate conditions were introduced which led to more and more missing responses. Moreover, it appeared that when a part of the central executive's capacity was allocated to a simultaneous card-sorting task, several aspects of the elderly's random generation ability were affected including monitoring abilities and also maybe shifting and coordination of storage functions. Indeed, in the dualtask condition, there were age-related differences for several measures of randomness and not only for the stereotype measure. In line with the idea of a reduced capacity of rapidly combine procedures, older adults seemed to be constrained to prioritize one of the tasks (random generation task) to the detriment of the other one (simultaneous card-sorting task), as observed with the numerous sorting errors.

Globally, the results could be interpreted as suggesting that the existence of a global reduction of the elderly's central executive resources (see also Van der Linden, Brédart, \& Beerten, 1994) along with a specific deficit affecting the ability to suppress natural well-learned response strategies. This reduced inhibition ability could be, at least partially, associated with the decline in frontal function which is considered to be a feature of normal aging (see Braun \& Lalonde, 1990; Moscovitch \& Winocur, 1992; Daigneault et al., 1993; West, 1996). 
However, this interpretation in terms of a reduction of the central executive resources and of a frontal deficit affecting inhibition has been questioned recently by Fisk and Warr (1996). Fisk and Warr (1996) compared older and younger subjects on a random generation task similar to that conducted in Experiment 1. They asked subjects to generate letters in a random sequence, at each of three production rates $(4,2$, and $1 \mathrm{~s})$. Only two measures of randomness were taken at each of the elicitation rates: the number of times any letter pair is repeated and the number of letter pairs which are alphabetically ordered (this yielded a total of six scores). Significant positive age correlations for five of the six scores were observed, which seems to indicate a decline of central executive function with age. In addition, the age effect in random generation (measured through one score which combined the preceding measures) remained significant after statistical control for the phonological loop functioning (measured through word span and digit span). However, these age differences in random generation were largely eliminated after controlling for age deficits in perceptual speed (measured by means of a letter and a pattern comparison speed task). These findings are consistent with the numerous studies conducted by Salthouse (for a review, see Salthouse, 1992; 1994) who showed that although increased age is associated with lower performance on working memory tasks, many of these agerelated differences appear to be mediated by a slower speed of processing. Therefore, it appears that the main part of the age-related variations observed in random generation could be due to a general reduction of perceptual speed rather than to a specific problem affecting the central executive. According to Salthouse (1996), reduction in speed leads to impairments in cognitive functioning because relevant operations cannot be successfully executed (the limited time mechanism) and because the products of early processing may no longer be available when later processing is complete (the simultaneity mechanism). In the context of random generation, slowing (through either limited time or simultaneity mechanisms) would disrupt the different operations of the central executive needed for random generation, that is to switch strategies, to access new strategies, and to monitor response output (Baddeley, 1996).

However, a speed interpretation of the age-related differences in random generation observed in the present study is confronted with different problems. First, if there exists a general speed factor which is responsible for the random generation deficits in the elderly, one may wonder why an age effect was not found on all measures of randomness in Experiment 1. In this context, it could be hypothesized that all components of random generation are not affected to the same extent by speed processing. Second, significant age differences (i.e., an increased number of stereotypes) were observed even in the very slow (4-s) rate condition. It could be argued that our elderly subjects were very slow and felt time pressure in a quite complex task. However, it could also be that processing speed was not the only factor involved in the 
decreased capacity of the elderly for generating random letter sequences and that other factors (especially, a reduction of the elderly's central executive resources and a deficit affecting inhibitory mechanisms) are also involved. In this perspective, it should be noted that Fisk and Warr (1996) did not examine the relative contribution of perceptual speed ability to random generation performance for each generation rate; rather, they combined the random generation measures at each elicitation rate into a composite score. Third, the speed interpretation has to explain the fact that in the dual-task condition, age differences were observed for most of the measures of randomness and not only for the stereotype score. It could be argued that in the dual-task condition, slowing disrupts the random generation performance of the elderly through both simultaneity and limited time mechanisms. However, it could also be that another factor (especially, a reduction of central executive resources) was responsible for the age-related differences. All these questions clearly need further investigation. Finally, it should be noted that we recently obtained data showing that the age-related differences observed on an updating working memory task which is considered to place significant demands on the central executive (Van der Linden et al., 1994; Salmon et al., 1996) could not be completely explained by variations in processing speed (Van der Linden, Beerten, \& El Ahmadi, 1996). These results suggest that specific deficits in the central executive can occur as a consequence of aging.

\section{APPENDIX}

TABLE A1

Summary of the Univariate and Stepdown Analysis of Variance for Experiment 1

\begin{tabular}{|c|c|c|c|c|c|}
\hline IV & DV & $\begin{array}{c}\text { Univariate } \\
\quad F\end{array}$ & $d f$ & $\begin{array}{c}\text { Stepdown } \\
F\end{array}$ & $d f$ \\
\hline \multirow{4}{*}{ Age } & Redundancy & 0.55 & $(1,30)$ & 0.6 & $(1,30)$ \\
\hline & Stereotypes & $4.1^{*}$ & $(1,30)$ & $4.15^{*}$ & $(1,29)$ \\
\hline & RNG index & 0.94 & $(1,30)$ & 0.99 & $(1,28)$ \\
\hline & Missed responses & $a$ & & $7.1^{*}$ & $(1,27)$ \\
\hline \multirow[t]{4}{*}{ Rate } & Redundancy & $29.3 * * *$ & $(2,60)$ & $29.3^{* * *} *$ & $(2,60)$ \\
\hline & Stereotypes & $28.9 * * *$ & $(2,60)$ & $21.1 * * *$ & $(2,59)$ \\
\hline & RNG index & $28.6 * * *$ & $(2,60)$ & 1.8 & $(2,58)$ \\
\hline & Missed responses & $7.9^{a * *}$ & & $5.1 * *$ & $(2,57)$ \\
\hline \multirow[t]{4}{*}{ Age $\times$ Rate } & Redundancy & 0.99 & $(2,60)$ & 0.99 & $(2,60)$ \\
\hline & Stereotypes & 1.06 & $(2,60)$ & 0.7 & $(2,59)$ \\
\hline & RNG index & 0.56 & $(2,60)$ & 0.1 & $(2,58)$ \\
\hline & Missed responses & $a$ & & $12.2 * * *$ & $(2,57)$ \\
\hline
\end{tabular}

Observed $p$ at least $*<.05, * *<.01, * * *<.0001$.

${ }^{a}$ As only older subjects missed responses, the univariate analysis on this DV was performed for this group only. 
TABLE A2

Pooled Within-Cell Correlations Among the Four $D V$ s (with standard deviations on diagonal) for Experiment 1

\begin{tabular}{lcccc}
\hline & Redundancy & Stereotypes & RNG index & Missed responses \\
\hline Redundancy & .019 & & & \\
Stereotypes & -.183 & 6.117 & & \\
RNG index & -.118 & .686 & .04 & 2.305 \\
Missed responses & .156 & -.388 & -.092 & \\
\hline
\end{tabular}

TABLE A3

Summary of the Univariate and Stepdown Analysis of Variance for Experiment 2

\begin{tabular}{llcccc}
\hline \multirow{2}{*}{ IV } & \multicolumn{1}{c}{ DV } & Univariate & \multicolumn{2}{c}{ Stepdown } & \\
& & $F$ & $d f$ & $\mathrm{~F}$ & $d f$ \\
\hline \multirow{2}{*}{ Age } & Redundancy & $4.3^{*}$ & $(1,30)$ & $4.3^{*}$ & $(1,30)$ \\
& Stereotypes & $13.2^{* *}$ & $(1,30)$ & $10.1^{* *}$ & $(1,29)$ \\
& RNG index & $6.43^{*}$ & $(1,30)$ & 0.4 & $(1,28)$ \\
& Missed responses & $a$ & & 1.2 & $(1,27)$ \\
Sorting & Sorting errors & $23.9^{* * *}$ & $(1,30)$ & $25.8^{* * *}$ & $(1,26)$ \\
& Redundancy & $9.73^{* * *}$ & $(3,90)$ & $9.7 * * *$ & $(3,90)$ \\
& Stereotypes & $2.9^{*}$ & $(3,90)$ & $3.8 *$ & $(3,89)$ \\
& RNG index & 1.6 & $(3,90)$ & 0.04 & $(3,88)$ \\
Age $\times$ Sorting & Missed responses & $2.1^{a}$ & $(3,45)$ & 2.1 & $(3,87)$ \\
& Sorting errors & $17.4^{* * *}$ & $(3,90)$ & $39.7 * * *$ & $(3,86)$ \\
& Redundancy & 1.3 & $(3,90)$ & 1.3 & $(3,90)$ \\
& Stereotypes & 0.8 & $(3,90)$ & 0.8 & $(3,89)$ \\
& RNG index & 0.5 & $(3,90)$ & 0.4 & $(3,88)$ \\
& Missed responses & $a$ & & 2.2 & $(3,87)$ \\
& Sorting errors & $11.8^{* * *}$ & $(3,90)$ & $33.7 * * *$ & $(3,86)$ \\
\hline
\end{tabular}

Observed $p$ at least $*<.05, * *<.01, * * *<.0001$.

${ }^{a}$ As only older subjects missed responses, the univariate analysis on this DV was performed for this group only.

TABLE A4

Pooled Within-Cell Correlations Among the Five DVs (with standard deviations on diagonal) for Experiment 2

\begin{tabular}{lrcccr}
\hline & Redundancy & Stereotypes & RNG index & $\begin{array}{c}\text { Missed } \\
\text { responses }\end{array}$ & $\begin{array}{c}\text { Sorting } \\
\text { errors }\end{array}$ \\
\hline Redundancy & .022 & & & & \\
Stereotypes & -.168 & 4.284 & & & \\
RNG index & .305 & .221 & .042 & & \\
Missed responses & .038 & -.189 & .032 & 1.175 & \\
Sorting errors & -.021 & -.167 & .005 & -.013 & 2.145 \\
\hline
\end{tabular}




\section{REFERENCES}

Attneave, F. 1959. Applications of information theory to psychology. New York: Holt, Rinehart, \& Winston.

Azouvi, Ph., Jokic, C., Van der Linden, M., Marlier, N., \& Bussel, B. 1996. Working memory and supervisory control after severe closed head injury. Journal of Clinical and Experimental Neuropsychology, 18, 1-21.

Baddeley, A. D. 1966. The capacity for generating information by randomization. The Quarterly Journal of Experimental Psychology, 18, 119-129.

Baddeley, A. 1996. Exploring the central executive. The Quarterly Journal of Experimental Psychology, 49A, 5-28.

Baddeley, A. D. 1986. Working memory. Oxford: Oxford University Press.

Bock, R. D., \& Haggard, E. A. 1968. The use of multivariate analysis of variance in behavioral research. In D. K. Whitla (Ed.), Handbook of measurement and assessment in behavioral sciences. Reading, MA: Addison-Wesley.

Braun, C. M. J., \& Lalonde, R. 1990. Les déclins des fonctions cognitives chez la personne âgée: Une perspective neuropsychologique. Canadian Journal of Aging, 9, 135-158.

Bray, J. H., \& Maxwell, S. E. 1985. Multivariate analysis of variance. Sage University paper series on quantitative applications in the social sciences, 07-054. Beverly Hills: Sage Publications.

Brugger, P., Monsch, A. U., Salmon, D. P., \& Butters, N. 1996. Random number generation in dementia of the Alzheimer type: A test of frontal executive functions. Neuropsychologia, 34, 97-103.

Brugger, P., Landis, T., \& Regard, M. 1992. The brain as a random generator: The relevance of subjective randomization for neuropsychology. Journal of Clinical and Experimental Neuropsychology, 14, 84 (abstract).

Daigneault, S., \& Braun, C. M. J. 1993. Working memory and the self-ordered pointing task: Further evidence of early prefrontal decline in normal aging. Journal of Clinical and Experimental Neuropsychology, 15, 881-895.

Daigneault, S., Braun, C. M. J., \& Whitaker, H. A. 1992. Early effects of normal aging on perseverative and nonperseverative prefrontal measures. Developmental Neuropsychology, 8, 99-114.

Deltour, J. J. 1993. Echelle de vocabulaire de Mill Hill de J. C. Raven. Adaptation française et normes européennes du Mill Hill et du Standard Progressive Matrices de Raven (PM 38). Braine-le-Château: Editions L'application des techniques modernes.

Evans, F. J. 1978. Monitoring attention deployement by random number generation: An index to measure subjective randomness. Bulletin of the Psychonomic Society, 12, 35-38.

Fisk, J., \& Warr, P. 1996. Age and working memory: The role of perceptual speed, the central executive, and the phonological loop. Psychology and Aging, 11, 316-323.

Moscovitch, M., \& Winocur, G. 1992. The neuropsychology of aging. In F. I. M. Craik and T. A. Salthouse (Eds.), The handbook of aging and cognition. Hillsdale, NJ: Erlbaum.

Petrides, M., \& Milner, B. 1982. Deficits on subject-ordered tasks after frontal and temporallobe lesions in man. Neuropsychologia, 20, 249-262.

Pollux, P. M. J., Wester, A., \& De Haan, E. H. F. 1995. Random generation deficit in alcoholic Korsakoff patients. Neuropsychologia, 33, 125-129.

Salmon, E., Van der Linden, M., Collette, F., Delfiore, G., Maquet, P., Degueldre, C., Luxen, A., \& Franck, G. 1996. Regional brain activity during working memory tasks. Brain, 119, 1617-1625. 
Salthouse, T. A. 1992. Mechanisms of age-cognition relations in adulthood. Hillsdale, NJ: Erlbaum.

Salthouse, T. A. 1994. The aging of working memory. Neuropsychology, 8, 535-543.

Salthouse, T. A. 1996. The processing-speed theory of adult age differences in cognition. Psychological Review, 3, 403-428.

Shimamura, A. P., \& Jurica, P. J. 1994. Memory interference effects and aging: Findings from a test of frontal lobe function. Neuropsychology, 8, 408-412.

Spatt, J., \& Goldenberg, G. 1993. Components of random generation by normal subjects and patients with dysexecutive syndrome. Brain and Cognition, 23, 231-242.

Spence, D. P., Wolitsky, D. L., \& Pezenik, J. 1969. Random generation of digits as a measure of attention: Relation to text redundancy and recall. Journal of Verbal Learning and Verbal Behavior, 8, 9-15.

Tabachnick, B. T., \& Fidell, L. S. 1989. Using multivariate statistics. New York: Harper \& Row.

Van der Linden, M., Brédart, S., \& Beerten, A. 1994. Age-related differences in updating working memory. British Journal of Psychology, 85, 145-152.

Van der Linden, M., Beerten, A., \& El Ahmadi, A. 1996. Effet du vieillissement sur la mise à jour de la mémoire de travail. Paper presented at the IVèmes Journées d'étude du Vieillissement Cognitif, november 1996, Louvain-la-Neuve, Belgium.

Wagenaar, W. A. 1972. Generation of random sequences by human subjects: A critical survey of literature. Psychological Bulletin, 77, 65-72.

West, R. L. 1996. An application of prefrontal cortex function theory to cognitive aging. Psychological Bulletin, 120, 272-292.

Wiegersma, S. 1982. Sequential response bias in randomized response sequences: A computer simulation. Acta Psychologica, 52, 249-256.

Wiegersma, S., \& Meertse, K. 1990. Subjective ordering, working memory, and aging. Experimental Aging Research, 16, 73-77. 